\title{
Regulatory gene networks that shape the development of adaptive phenotypic plasticity in a cichlid fish
}

\author{
RALF F. SCHNEIDER, ${ }^{*} \dagger$ YUANHAO LI,$*$ AXEL MEYER*† and HELEN M. GUNTER $\ddagger^{*}$ \\ *Lehrstuhl für Zoologie und Evolutionsbiologie, Department of Biology, University of Konstanz, Universitätstrasse 10, 78457 \\ Konstanz, Germany, †International Max Planck Research School for Organismal Biology, University of Konstanz, \\ Universitätsstr 10, 78457 Konstanz, Germany, \$Zukunftskolleg, University of Konstanz, Universitätsstr 10, 78457 Konstanz, \\ Germany
}

\begin{abstract}
Phenotypic plasticity is the ability of organisms with a given genotype to develop different phenotypes according to environmental stimuli, resulting in individuals that are better adapted to local conditions. In spite of their ecological importance, the developmental regulatory networks underlying plastic phenotypes often remain uncharacterized. We examined the regulatory basis of diet-induced plasticity in the lower pharyngeal jaw (LPJ) of the cichlid fish Astatoreochromis alluaudi, a model species in the study of adaptive plasticity. Through raising juvenile $A$. alluaudi on either a hard or soft diet (hard-shelled or pulverized snails) for between 1 and 8 months, we gained insight into the temporal regulation of 19 previously identified candidate genes during the early stages of plasticity development. Plasticity in LPJ morphology was first detected between 3 and 5 months of diet treatment. The candidate genes, belonging to various functional categories, displayed dynamic expression patterns that consistently preceded the onset of morphological divergence and putatively contribute to the initiation of the plastic phenotypes. Within functional categories, we observed striking co-expression, and transcription factor binding site analysis was used to examine the prospective basis of their coregulation. We propose a regulatory network of LPJ plasticity in cichlids, presenting evidence for regulatory crosstalk between bone and muscle tissues, which putatively facilitates the development of this highly integrated trait. Through incorporating a developmental time-course into a phenotypic plasticity study, we have identified an interconnected, environmentally responsive regulatory network that shapes the development of plasticity in a key innovation of East African cichlids.
\end{abstract}

Keywords: Astatoreochromis alluaudi, bone remodelling, cichlid, developmental time-course, evodevo, pharyngeal jaw

\section{Introduction}

Traditionally, research on adaptive morphological variation has focused predominantly on genetic variability (e.g. Clausen et al. 1941; Morgan 1947; Lewontin 1974). However, throughout recent decades, investigations

Correspondence: Axel Meyer, Fax: +49 (0) 753188 3018; E mail: axel.meyer@uni konstanz.de

${ }^{1}$ Present address: Edinburgh Genomics, Ashworth Labora tories, University of Edinburgh, West Mains Road, Edinburgh, EH9 3JT, UK have demonstrated that a broad range of environmental variables, both internal and external to the organism, play an instructive role in the development of adaptive phenotypes (Adler \& Harvell 1990; Janzen \& Paukstis 1991; West-Eberhard 2004; Gilbert 2005; Beldade et al. 2011). Phenotypic variability can arise when environmental cues modulate the developmental trajectories encoded by a single genotype through a process termed phenotypic plasticity (Schlichting \& Pigliucci 1998; Pfennig et al. 2010). Integrative approaches such as 'ecological developmental biology', which incorporate evolutionary biology, developmental biology and ecol- 
ogy, have the power to shed new light on the role of phenotypic plasticity in evolution (Gilbert 2001; WestEberhard 2004; Gilbert \& Epel 2009). While a historical lack of strong molecular evidence has hampered the acceptance that phenotypic plasticity would promote (rather than impede) speciation, logically it stands to reason that it should promote speciation under certain ecological circumstances (Price et al. 2003; Nicotra et al. 2010). Indeed, one of the basic concepts of evolutionary biology is that selection acts on an organism's phenotype (Mayr 1963; Lande \& Arnold 1983; Schwander \& Leimar 2011; Scheiner \& Holt 2012) regardless of whether this phenotype is genetically encoded, opening the possibility that plastic phenotypes might represent an important intermediate step in speciation. Moreover, it has been recognized that phenotypic plasticity itself can be the target of selection (Pigliucci 2007; Wund et al. 2008).

In spite of its potential importance in generating adaptive phenotypes, understanding the molecular mechanisms underlying phenotypic plasticity has only become technically possible in recent years. To date, several ground-breaking studies have shown that alternative phenotypes may be induced through the alteration of gene expression by epigenetic means, as well as alterations of hormonal and enzymatic activity (Denver 1997; Gilbert 2005; Aubin-Horth \& Renn 2009; Jablonka \& Raz 2009; Snell-Rood et al. 2010; Beldade et al. 2011; Sommer \& Ogawa 2011). Such studies typically focus on the molecular bases of polyphenisms, discrete alternative phenotypes that arise through environmentally mediated switches in developmental pathways (Abouheif \& Wray 2002). Nonetheless, trait values for environmentally induced phenotypes often form a continuous distribution, the reaction norm (Woltereck 1913).

To determine the molecular bases of phenotypic plasticity, as a crucial step to evaluating its effects on species evolution, it is essential to investigate the temporal expression dynamics of environmentally responsive genes that mediate the plastic response, for which developmental time-course experiments are a powerful tool (Aubin-Horth \& Renn 2009). For organisms that respond plastically to an environmental cue, genes that are upstream in a regulatory cascade underlying a plastic trait are expected to alter their expression earlier in the presence of this inductive cue compared to genes that are more downstream (Aubin-Horth \& Renn 2009). Thus, it is necessary to interpret changes in gene expression in a plastic trait throughout development, to be able to delineate the roles of specific genes throughout the plastic response.

In a previously published analysis, we characterized the molecular basis of diet-induced phenotypic plasticity in the lower pharyngeal jaw (LPJ) in an East African fish, Astatoreochromis alluaudi (Gunter et al. 2013). The LPJ forms part of the pharyngeal jaw apparatus (PJA), a key innovation of cichlid fishes that contributed significantly to their explosive adaptive radiation within $<2$ Myr (Liem 1974; Meyer et al. 1990; Elmer et al. 2009). Through evolving highly specialized feeding morphologies and behaviours, many East African cichlids have been able to exploit narrow and creative trophic niches, such as algae scraping, Aufwuchs plucking and insect picking, often in parallel across the three main African lakes (Meyer et al. 1990; Kocher et al. 1993; Meyer 1993a; Stiassny \& Meyer 1999; Schön \& Martens 2004; Young et al. 2009). In contrast, A. alluaudi is a generalist species, basal to the modern haplochromines, which inhabits Lake Victoria, its satellite lakes and associated river systems (Greenwood 1964; Hoogerhoud 1984; Salzburger et al. 2005). Its' plastic LPJ allows this cichlid to exploit the available food resources efficiently across varying habitats: if their preferred diet of soft food (such as insects) is sufficiently available, they develop a slender, 'papilliform' LPJ, bearing numerous fine teeth (Slootweg et al. 1994). However, if soft food is scarce, individuals feed on hard-shelled molluscs that induce the formation of a robust molariform LPJ, bearing fewer, more molar-like teeth (Fig. 1) (Greenwood 1964; Huysseune et al. 1994; Slootweg et al. 1994; Huysseune 1995). Further cases of trophic plasticity have been investigated amongst Neotropical cichlids, leading to the hypothesis that these might be more phenotypically plastic than most African cichlids (Meyer 1987, 1989, 1990, 1993b; Muschick et al. 2011). However, the general relationship between phenotypic plasticity and speciation rates is still unclear and remains hotly debated (Pfennig et al. 2010; Thibert-Plante \& Hendry 2011; Landry \& Aubin-Horth 2014).

In this study, we investigate the molecular basis of environmentally induced morphological divergence that results in the papilliform and molariform LPJ phenotypes in A. alluaudi. A time-course experiment was employed, exposing naïve juvenile fish to experimental diets for variable time periods, allowing us to analyse gene expression across 8 months of plastic development in A. alluaudi. We investigated the expression of 19 candidate genes that were previously found to be associated with induced plasticity in the LPJs of A. alluaudi (Gunter et al. 2013). Of these 19 candidates, 16 belong to six main functional categories, including 'immediate early genes', 'haem pathway genes', 'matrix-related genes', 'bone-related genes', 'muscle-related genes' and 'calcium pathway genes '. We present evidence that most of these genes are not only associated with the plastic phenotypes, but that they also contributed to the early and on-going development of LPJ plasticity in A. alluaudi. Finally, we shed light on the putative 

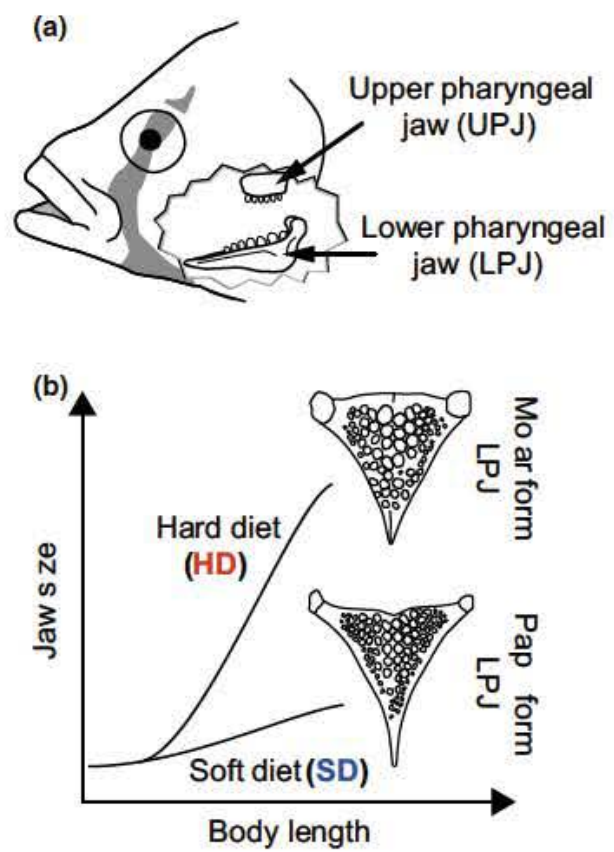

Fig. 1 Cichlid fishes possess a mechanically robust pharyngeal jaw apparatus. (a) The PJA is comprised of a pair of upper pharyngeal jaws (UPJ) that articulate directly with the neuroc ranium and the lower pharyngeal jaw (LPJ), which is formed by the suturing of the 5 th ceratobranchial arches. (b) For the cichlid Astatoreochromis alluaudi, the mechanical properties of the diet influence LPJ development, whereby individuals fed a soft diet develop a smaller, more slender papilliform LPJ, the baseline condition, while individuals fed a hard diet develop a larger, more robust molariform LPJ that withstands increased biting forces. Image modified from Hoogerhoud (1984).

regulatory network underlying LPJ plasticity by utilizing an analysis of transcription factor binding sites on the genome of a closely related cichlid.

\section{Materials and methods}

\section{Fish husbandry and experimental set-up}

A developmental time-course experiment was conducted on an inbred strain of Astatoreochromis alluaudi using modifications to the methods described in Gunter et al. (2013), assuring minimal genetic variation between individuals. Two broods (which share the same grandparents) comprising a total of $\sim 40$ individuals were raised on Artemia salina nauplii and Tetramin flake food until they reached a standard length (SL) of $\sim 30 \mathrm{~mm}$. These individuals were split randomly in two equally sized groups, and each group was raised in a substratefree 100-1 tank for up to 8 months. Tanks were set up in a temperature-controlled room set to $25^{\circ} \mathrm{C}$ with artificial illumination, regular water changes and weekly water quality assessments ensuring minimal heterogeneity between the tanks. Comparisons to an independent study with a similar experimental design (H. M. Gunter unpublished results) demonstrated that there were no systemic tank effects (Fig. S1, Supporting Information). Each of the two groups was fed Melanoides spp. snails, with one group receiving whole snails, which the fish had to crack with their pharyngeal jaw apparatus (PJA) (the hard diet (HD) group), and the second group receiving an equivalent amount of snails that had been finely pulverized (the soft diet (SD) group). After 1, 3, 5 and 8 months of treatment, fish from each of the two groups were randomly selected and sacrificed within $30 \mathrm{~min}$ of feeding using a lethal dose of Tricaine (MS222). These time points were chosen to evenly cover the treatment period, which was predicted to include size of plastic divergence, previously determined to be $55 \mathrm{~mm}$ SL (Huysseune et al. 1994). Mean SL did not differ between the two diet groups for any of the four developmental time points (Fig. S2, Supporting Information). A total of 33 individuals were sampled across all developmental stages. Sex was not taken into account as previous investigations indicated that diet and SL are far greater determinants of LPJ plasticity in A. alluaudi (Gunter et al. 2013). The fish's SLs were measured, LPJs were dissected and stored in RNAlater (Qiagen) at $20^{\circ} \mathrm{C}$.

\section{Morphometric measurements}

To determine the stage at which LPJ plasticity could first be detected, the size of each LPJ was analysed according to Gunter et al. (2013). These analyses were made from digital images, which were captured after the LPJs had been cleaned of surrounding connective tissue. Ten linear morphometric measurements of all LPJs were obtained following Gunter et al. (2013) (Fig. S3, Supporting Information). ANCOVAs were performed using R (RDC-Team 2005), utilizing each linear morphometric measure as a dependent variable, diet as a factor and SL as a covariate (Fig. S4, Supporting Information). SL was plotted against each linear measurement to evaluate the relationship between size and morphological divergence. As there was a significant linear relationship between SL and all other linear morphometric measurements (for linear regressions all $P<0.05$ ), only the residuals of the respective variables were used for further analyses. The morphological differences amongst the diet groups were evaluated for each time point by performing Wilcoxon signed-rank tests in R. Multiple testing correction was not performed, but we chose rather to interpret our results with caution. All $P$ values are listed in the Supporting Information (Tables S2,S3, Supporting information). 


\section{RNA extraction and $c D N A$ synthesis}

RNA was extracted from the LPJ samples using the RNA Mini kit (Qiagen). In addition to the steps recommended by the manufacturer, LPJs were ground to a fine powder while submerged in liquid nitrogen and homogenized in a FastPrep (MP Biomedicals). Polyacryl carrier (MRC) was added to increase RNA yield, and samples were treated with an optional on-column DNase treatment (Qiagen). Furthermore, additional wash steps with $80 \%$ ethanol were included to remove all traces of salt. RNA quantity was assessed using a fluorometer (Qbit 2.0), and its integrity was confirmed using a Bioanalyzer 2100 (Agilent). RNA was determined to be free of gDNA contamination according to noRT controls, and cDNA was synthesized using Invitrogen SuperscriptIII and oligo dT primer, according to Gunter et al. (2013).

\section{qRT-PCR}

We examined the expression of 19 candidate genes that were previously shown to be either up- or downregulated in the LPJs of $A$. alluaudi individuals that received a hard diet (HD), vs. the control, which received a soft diet (SD), for a period of 18 months (Gunter et al. 2013). The upregulated genes include: cfos, ier2 and rgs2, termed immediate early genes (Nose \& Shibanuma 1994; Versele et al. 1999; Ott et al. 2009); klf4, a pleiotropic transcription factor involved in differentiation of stem cells including osteoblasts and osteoclasts (Nose \& Shibanuma 1994; Michikami et al. 2012); ryr1, anxa6 and srl, termed calcium pathway genes (Leberer et al. 1989; Meissner 1994; Song et al. 2002; Treves et al. 2005); tnnt, tpm4 and des, termed muscle-related genes (Perry 1998; Mantila Roosa et al. 2011); col6, col12 and thbs3, termed matrix-related genes (Tucker et al. 1997; Gelse et al. 2003) and run $2 b$ and os $x$, which orchestrate osteoblast proliferation and differentiation, termed bone-related genes (Nakashima et al. 2002; Li et al. 2009b). Downregulated genes include alas1 and c1ql, which are putatively associated with haem biosynthesis and haematopoiesis, termed haem pathway genes (Sadlon et al. 1999; Mei et al. 2008); abcb3 and gif, which are associated with MHC function (Edidin 1983; Karttunen et al. 2001; Nonaka \& Nonaka 2010) and Vitamin B12 binding and transport, respectively (Greibe et al. 2012) (Table S1, Supporting Information).

Primers used in this study were either described previously in Gunter et al. (2013) or were derived from RNA-seq contigs generated by the same study, and their efficiency was tested using standard curves using a Bio-Rad CFX96 cycler (efficiencies ranged between 92\% and 108\%; Table S1, Supporting Information). As in Gunter et al. (2013), actinr and twinfilin were used as housekeeping genes, which were selected from an RNA-seq data set, on the basis of showing the lowest variability between diet treatments and individuals. Primer concentrations and annealing temperatures were optimized to ensure negligible dimer amplification, based on assessment of the melt curve and comparison to no template controls. All primers were used at a concentration of $0.3 \mathrm{pmol} / \mu \mathrm{L}$, and annealing temperatures were $60{ }^{\circ} \mathrm{C}$ for ier2, gif and klf4 and $55{ }^{\circ} \mathrm{C}$ for all other genes. For each gene, all samples were run in duplicate on a single 96-well plate, alongside a no template control. For each run, we ensured that negligible/no dimer was produced based on assessment of the melt curve and comparison to the no template controls. Gene expression values were calculated for each sample and gene using the respective primer efficiencies. Relative quantification values (RQ-values) were calculated by scaling to the maximum expression value observed for each gene. Relative quantification values were further normalized to a normalization factor, calculated from the expression of the aforementioned housekeeping genes (Vandesompele et al. 2002).

\section{Statistical analysis of $q R T-P C R$}

Wilcoxon signed-rank tests were performed to analyse differences in candidate gene expression between diet groups across the four time points as data distributions were nonparametric. We did not perform correction for multiple testing (see above) and report all $P$-values in the Supporting Information (Table S4, Supporting Information). A PCA was performed on a covariation matrix to explore patterns of co-expression within our gene expression data set. A hierarchical cluster analysis was then conducted using a Spearman rank correlation based matrix for specimen clustering and Euclidean distances for gene expression clustering in R, to independently examine the robustness of any identified clusters and to explore the clustering of specimens across treatment and diet groups (following Haas et al. (2013)). Throughout the study, we applied a significance level of $\alpha \quad 0.05$.

\section{Analysis of transcription factor binding sites}

To gain insight into the regulatory network of LPJ plasticity, we investigated the distribution of transcription factor binding sites (TFBSs) in the promoter regions of the candidate genes. As the genome of $A$. alluaudi has not been sequenced, the genome of the closely related cichlid Astatotilapia burtoni was chosen for this purpose. Both species are generalist feeders, inhabiting comparable trophic niches, and phenotypic plasticity can be induced by diet in the LPJs of each, although to a lesser 
extent in A. burtoni (H.M. Gunter, unpublished). Moreover, they display a high degree of genetic similarity, computed to be $99.13 \%$ based on exon similarity calculations performed using samtools. To calculate this similarity, we divided the total number of SNPs between the two species by the total number of aligned nucleotides (indels were masked samtools-0.1.18; Li et al. 2009a).

We identified all 19 candidate genes in the genome of A. burtoni and used Jaspar to investigate the distribution of TFBSs in their promoters (version 5.0; http:// 129.177.120.189/cgi-bin/jaspar2010/jaspar db.pl). The investigated interval spanned from $1 \mathrm{~kb}$ upstream from each gene's translation start site to the end of the first exon, encompassing both the putative promoter region and the $5^{\prime}$-UTR (similar to Lerch et al. (2012)). In some cases (cfos, rgs2, runx $2 b$, alas1 and anxa6), more than one translation start site was identified, and we analysed the promoters of each separately, accounting for potential overlaps. TFBSs were selected above a conservative threshold of 0.85 (Kwon et al. 2012) of the relative matrix score, and results for threshold levels of 0.9 and 0.95 are also reported as an indicator of the distribution of TFBS qualities. Moreover, we focused on the distribution of binding sites for two transcription factors that have been shown to be mechanically responsive: AP1 (a heterodimer comprising a JUN and a FOS protein unit, referred to as 'JUN::FOS' in Jaspar) and CREB1 (cAMP response element-binding protein 1) (Davidovitch et al. 1984; Nomura \& Takano-Yamamoto 2000). Finally, we examined TFBSs for RUNX2B ('RUNX2' in Jaspar) and KLF4 to identify further potential regulatory pathways amongst our candidate genes.

\section{Results}

Diet influences morphological divergence in Astatoreochromis alluaudi LPJ development

Using a split brood experiment, we investigated the regulatory networks underlying the development of diet-induced adaptive plasticity in the LPJ of Astatoreochromis alluaudi. Our analyses indicated the on-going divergence of LPJ size between diet groups across the developmental time-course, with the majority of significant differences being detected after 5 and 8 months of treatment (Fig. 2, Table S2, Fig. S3, Supporting Information). At these time points, HD fish already displayed the typical molariform LPJ phenotype, with relative increases in jaw weight, average tooth size, centroid size and size of muscle attachment horns, in comparison with SD fish.

The ANCOVA analyses on the linear morphometric data indicated a significant factor effect and a significant interaction for the log of jaw weight and suture width, suggesting morphological divergence between the two diet groups. In addition, average tooth size and centroid size also showed increased values in the HD treatment, and although they did not attain statistical significance, they displayed a trend towards morphological divergence $(0.05<P<0.1)$ between diet treatments (Fig. 2). Indeed, this trend was observed for most linear measurements and was supported by nonoverlapping confidence intervals (Table S3, Fig. S4, Supporting Information).

Our linear morphometric data show that morphological divergence in the LPJ of A. alluaudi was first detectable at 5 months of treatment. The confidence intervals of our scatterplots suggest that significant morphological divergence is likely to have occurred at a SL of $5560 \mathrm{~mm}$ (which coincides with a treatment duration of between 3 and 5 months, Fig. S2, Supporting Information) for variables such as LPJ weight, suture width and average horn width (Fig. 2, Fig. S4, Supporting Information). Such variables are indicative of a robust, functionally integrated molariform LPJ phenotype, where the LPJ is denser, the two 5th ceratobranchials are united by a stronger suture, and movement of the LPJ is controlled by larger, stronger muscles (e.g. Huysseune et al. 1994; Smits et al. 1996; Hulsey 2006). This divergence lies in the middle of the developmental time-course employed by our study, enabling gene expression to be compared for stages prior to and after the onset of observable morphological divergence.

\section{Diet influences gene expression during Astatoreochromis alluaudi LPJ development}

Quantitative reverse transcription PCR (qRT PCR) was used to analyse the expression of previously identified candidate plasticity genes in a developmental time-course of diet-manipulated A. alluaudi. As determined by our morphological analyses, this time-course captured the period prior to and shortly following the development of observable morphological differences that arose due to the plastic response. We observed significant gene expression differences between treatments after 3,5 or 8 months of treatment (rgs2, ryr1, anxa6 and tnnt; osx and alas1; col12, respectively) (Fig. 3, Table S4, Supporting Information). Further trends of differential expression $(0.1>P>0.05)$ were observed after 3,5 or 8 months of treatment ( $\mathrm{srl}$, tpm4 and des; tpm4 and gif; srl and os $x$, respectively). After 1 month of treatment, mean expression levels were higher in SD than HD individuals for 17 of the 19 candidate genes (albeit not significantly); however, this pattern was inverted for most genes after 3 months of treatment (Fig. 3, Table S4, Supporting Information). After treat- 

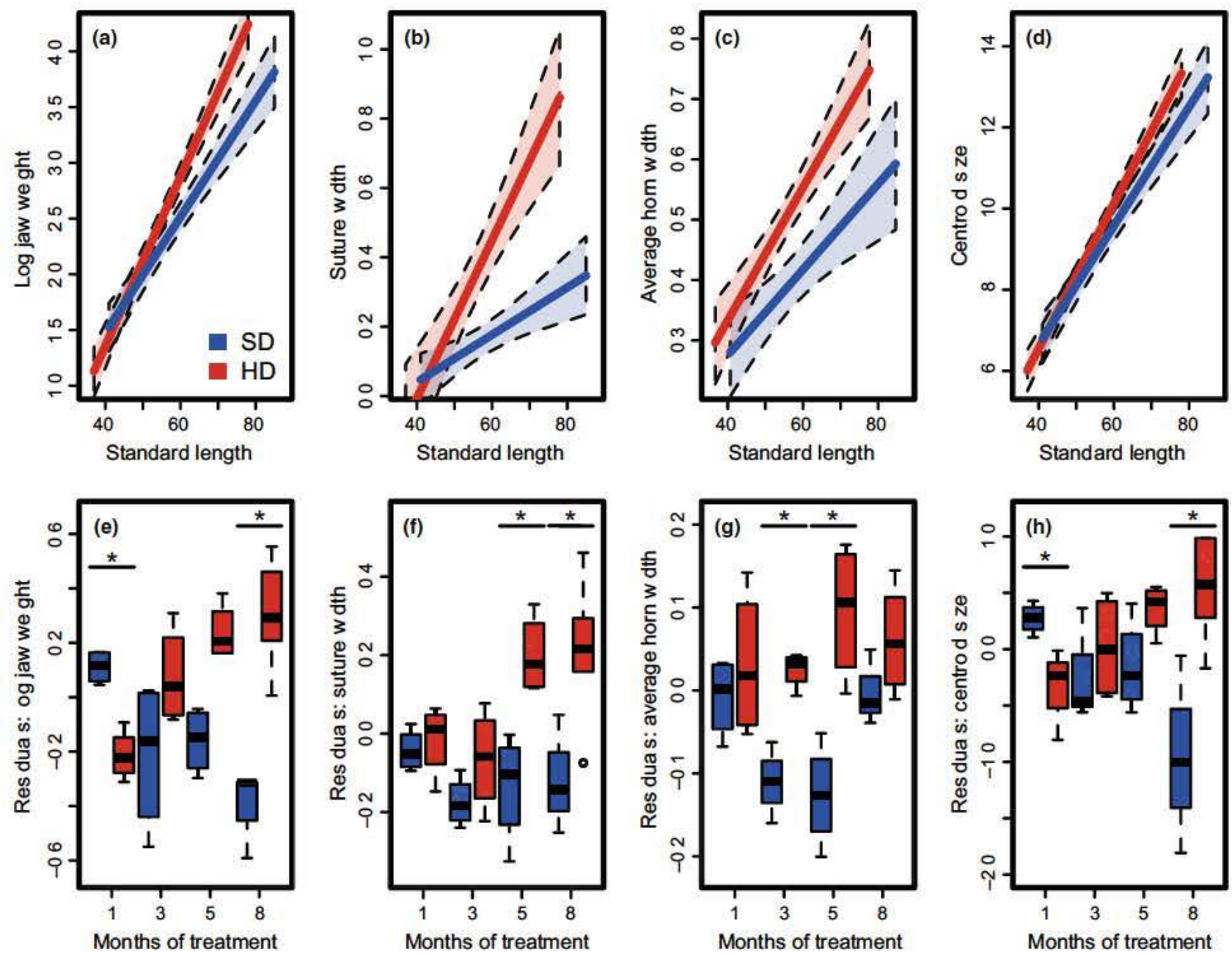

Fig. 2 Developmental plasticity in LPJ size for Astatoreochromis alluaudi fed either a hard diet (HD) or soft diet (SD). Four linear mor phometric measurements were plotted against (a d) standard length and (e h) months of treatment. Morphometric measures include $(a, e)$ LPJ weight, $(b, f)$ suture width, $(c, g)$ horn width and $(d, h)$ centroid size. (a d) Different slopes were detected for each morpholog ical measurement in each diet group, indicating that morphological divergence arose at $\sim 5560 \mathrm{~mm}$. (e h) Residuals of morphometric measurements tended to increase in HD compared with SD individuals across development $\left(^{*}=P<0.05\right)$. Marked areas reflect $95 \%$ CIs ( $\mathrm{a} \mathrm{d}$ ) and boxplots show the median, the 1st and 3rd quartiles as hinges and upper and lower whiskers (e h).

ment periods of 5 and 8 months, consistent trends were not observed amongst all genes, but we observed divergent gene expression patterns between the functional categories. Within functional categories, we observed a high degree of co-expression across most genes when diet-specific expression patterns were examined across all developmental time points. Additional analyses were thus employed to formally explore the degree of co-expression within the functional categories, including a principal component analysis (PCA) and hierarchical clustering.

\section{Genes within functional categories display marked co-expression}

PCA was used to analyse co-expression between the candidate genes, comparing gene expression values from $\mathrm{HD}$ and SD treatments across all developmental time points. Groups of co-expressed genes were identified through visual examination of the PCA plots (Fig. 4, Table S6, Supporting Information). Three groups were clearly identifiable in each of the first three principle components (PCs) of the analysis, namely (i) the muscle-related genes and srl clustered on PC1; (ii) immediate early genes and klf4 aligned on $\mathrm{PC} 2$; and (iii) matrix-related genes aligned on PC3. Additional genes grouped more loosely on the first three PCs. Specifically, anxa6 and ryr1 were associated with both the musclerelated genes on $\mathrm{PC} 1$, and the matrix-related genes on PC 3 . Also, the bone-related genes run $x 2 b$ and os $x$ aligned with the matrix-related genes on $\mathrm{PC} 3$, albeit less of their total variance was explained by this PC 


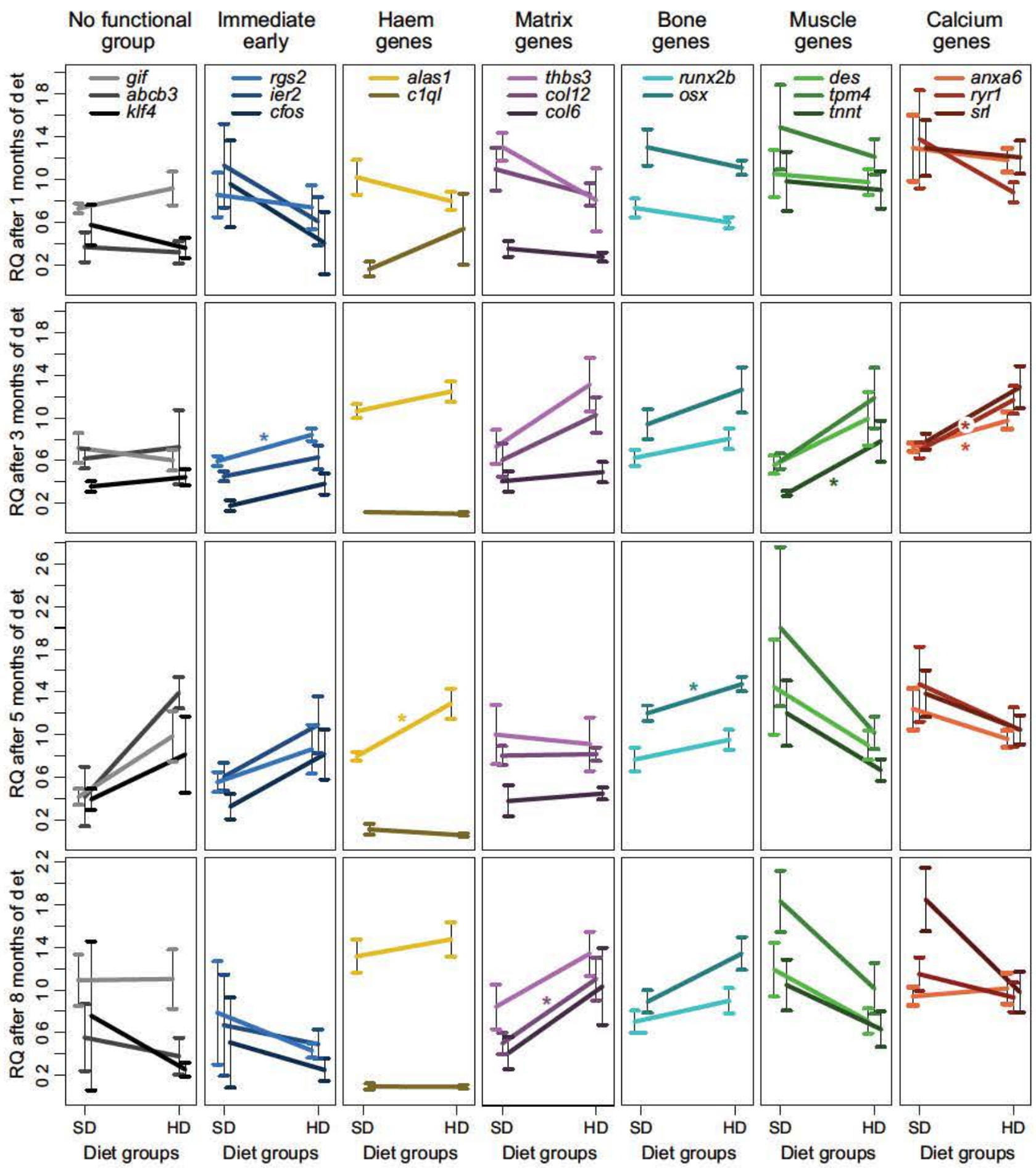

Fig. 3 Expression of candidate genes displays a dynamic pattern during the development of diet induced plasticity in the LPJ. Reac tion norms of gene expression for SD vs. HD treatments, with expression plotted separately for treatment periods of $1,3,5$ and 8 months, and for genes of each functional category. Genes within functional categories tend to display similar patterns of expression within each time point, while expression is highly differential between developmental stages, and between functional categories (* $\quad P<0.05)$. Shared line coloration hues reflect the respective functional categories.

than for the matrix-related genes. Moreover, a few genes did not display detectable co-expression, namely the haem pathway genes, in addition to $a b c b 3$ and gif. No further meaningful co-expression groups could be identified (Table S6, Supporting Information). 
A hierarchical cluster analysis was then conducted, using only the genes that displayed clustered co-expression according to the PCA (Fig. 5, Fig. S1, Fig. S6, Supporting Information). Similar to the PCA, three distinct
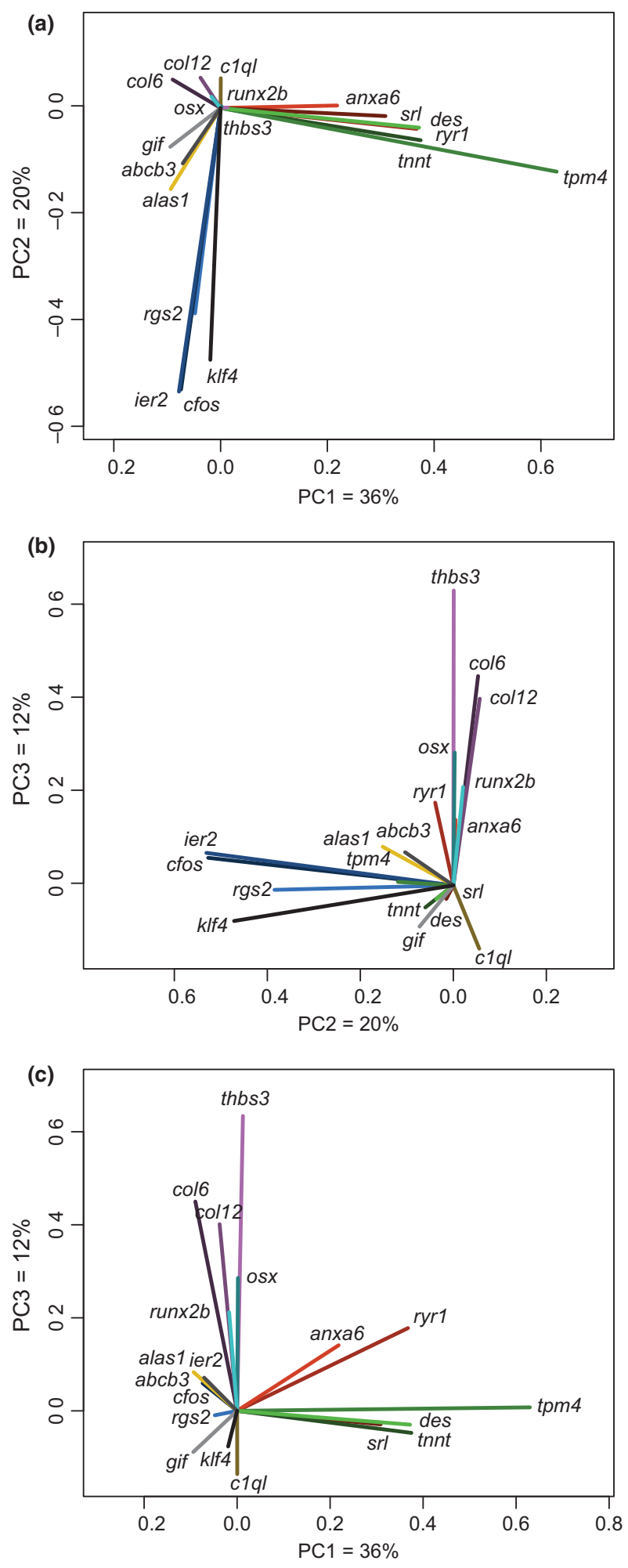

clusters were detected, with gene compositions that concurred with results of the PCA. Immediate early genes and klf4 formed one cluster, calcium pathway genes clustered with the muscle-related genes and the bone-related genes clustered with the matrix-related genes, suggesting that these clusters form three putative regulatory modules. When all expression patterns were considered, specimens clustered neither according to their treatment group nor the treatment duration, suggesting that the treatment effects were rather subtle in our samples, consistent with our observation of subtle morphological differences.

Transcription factor binding site analysis indicates regulatory basis of co-expression

A transcription factor binding site (TFBS) analysis was utilized to evaluate the regulatory basis of the identified patterns of co-expression amongst functional categories. Using the TFBS detection program Jaspar, a total of 10,741 TFBSs were identified in the promoter regions and $5^{\prime}$-UTRs of our 19 candidate genes. Of these, 244 were binding sites of four key TFs that are putatively involved in establishing the observed plastic phenotypes. These include two mechanically responsive TFs, AP1 and CREB1, in addition to two bone-related TFs KLF4 and RUNX2B (Fig. 6, Fig. 7, Table S7, Supporting Information).

We estimated the strength of regulatory relationships between the selected TFs and our candidate genes, based on the total number of predicted TFBSs in their promoters, and the predicted binding strength at each TFBS (based on the position weight matrix score) (Hallikas et al. 2006). Based on this, the strongest putative regulatory targets of AP1 were estimated to be the immediate early genes cfos, rgs2 and ier2, both collagens and run $2 b$ and des. Moreover, AP1 was estimated to have a more moderate regulatory influence on $a b c b 3$, alas1, c1ql, gif, os $x$ and the remaining muscle-related and calcium pathway genes. We estimated the strongest putative regulatory target of CREB1 to be runx2b, while having a more moderate influence on cfos, rgs2, klf4, srl

Fig. 4 PCA on candidate gene expression patterns indicates coregulation amongst functionally related genes. $(a, c)$ Muscle related and calcium pathway genes cluster on PC1, which explains $36 \%$ of the total variation. (a,b) Immediate early genes and klf4 cluster on PC2, which explains $20 \%$ of the total varia tion. (b,c) Matrix and bone related genes cluster on PC3, which explains $12 \%$ of the total variation. Plot lines of the PCA are colour coded according to the functional class of each gene: immediate early genes are blue, calcium pathway genes are red, muscle related genes are green, matrix related genes are violet, bone related genes are cyan, haem pathway genes are ochre and others black (as in Fig. 3). 


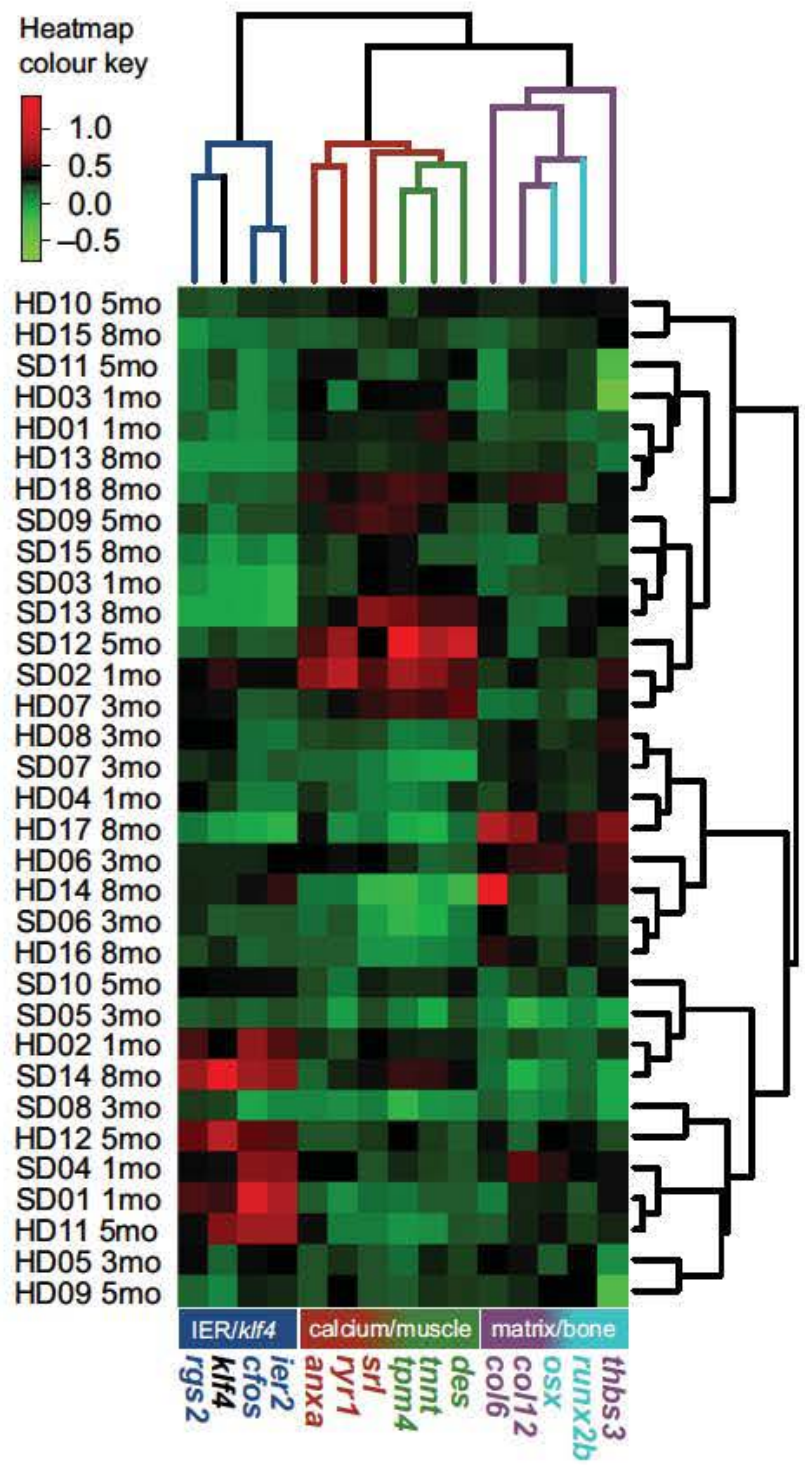

Fig. 5 Hierarchical cluster analysis on candidate gene expres sion patterns indicates coregulation amongst functionally related genes. The analysis shows three main clusters: (i) klf4 and the immediate early genes, (ii) bone related genes and matrix related genes and (iii) calcium pathway genes and mus cle related genes. HD hard diet, mo months of treatment, SD soft diet. Coloration of the gene names refers to their respective functional class: immediate early genes are blue, cal cium pathway genes are red, muscle related genes are green, matrix related genes are violet, bone related genes are cyan and others black (as in Fig. $3 \& 4$ ).

and tnnt. RUNX2B was estimated to have a moderate regulatory influence on gif, runx $2 b$ and os $x$. Finally, KLF4 was estimated to have the strongest regulatory influence on alas 1 and a more moderate influence on c1ql, rgs2, klf4, osx, anxa6, ryr1, tnnt and des.

Of the 170 TFs for which binding sites were detected, twelve were shared amongst all candidate genes.

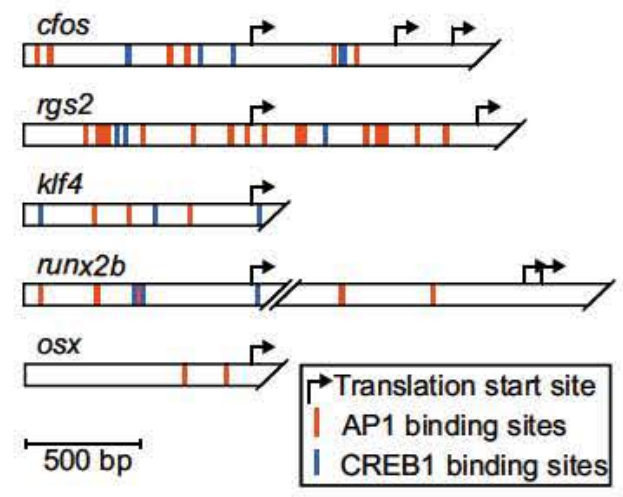

Fig. 6 The regulatory regions of our candidate genes contain numerous predicted binding sites for mechanically responsive TFs. Coloured lines indicate predicted binding sites for the mechanically responsive transcription factors AP1 and CREB1 (cAMP response element binding protein 1) in the regulatory regions of cfos, rgs2, klf4, run $x 2 b$ and $o s x$. Immediate early genes cfos and rgs 2 include the highest density of mechanically respon sive TFBSs.

Within functional categories, we detected characteristic TFBSs that may contribute to the observed co-expression. Amongst all immediate early genes and klf4, binding sites for 14 TFs were detected that were not shared by all members of any other functional group. These include the mechanically responsive TFs JUN, GATA4 and CEBP $\beta$ (Sumpio et al. 1994; Swynghedauw 2006; Sen et al. 2009). For all matrix and bone-related genes, binding sites for three TFs were detected. For all muscle-related and calcium pathway genes, binding sites for two TFs were detected, including the muscle-specific TF MYOG that contributes to myogenic cell differentiation in mesenchymal stem cells (Wright et al. 1989). Binding sites for two TFs (MYCN and FOS) were detected in the calcium pathway genes ryr1 and anxa6, but not in any other muscle-related gene or $s r l$, potentially explaining their relatively distinct expression in comparison with the other genes within their co-expression cluster.

Interestingly, amongst TFs with the most abundant binding sites in the promoter regions of our candidate genes, there are two that are known to be involved in development and plasticity, which thus may have contributed to the observed plastic phenotypes (Table S7, Supporting Information). These include ARID3A, which is involved in the regulation of developmental plasticity in mouse and human cells (An et al. 2010) and PRRX2, which is involved in craniofacial bone development in mice (Lu et al. 1999) as well as matrix alterations of the vascular system and smooth muscles (Bergwerff et al. 1998). Binding sites for both TFs were found to be present in high numbers in the promoter of $o s x$, a major regulator of bone development. 


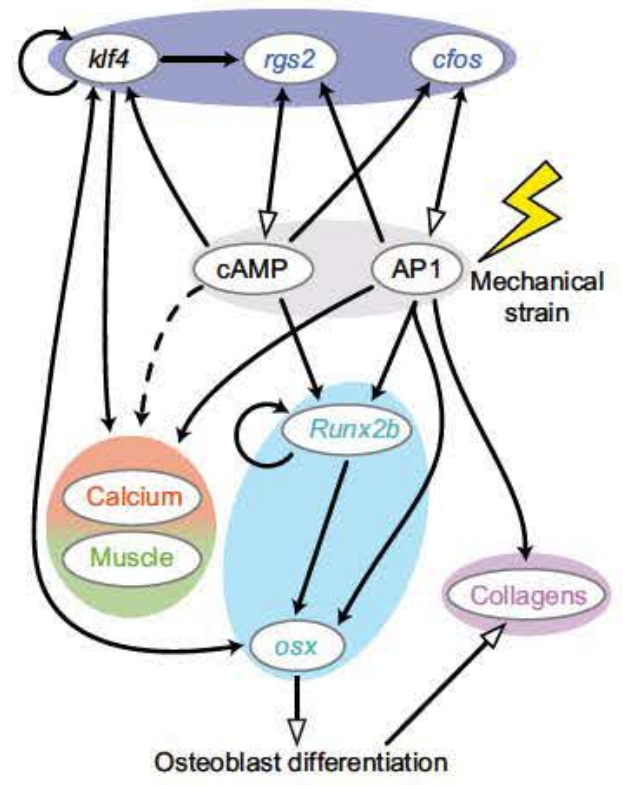

Fig. 7 Major regulatory pathways of LPJ phenotypic plasticity as suggested by the TFBS analysis. Closed arrowheads indicate the direction of regulatory influence inferred from the analysis, while open heads indicate regulatory relationships described in previous publications (see text for details). The dashed arrow targeting 'calcium pathway' and 'muscle related' genes indicates that only some, but not all of these genes exhibit CAMP (mediated by CREB1) TFBSs. The strain responsive tran scription factor AP1 appears to be of central role in the upstream regulation of LPJ phenotypic plastic response to mechanical strain, regulating collagens, calcium and muscle related genes, immediate early genes and runx $2 b$. run $x 2 b$ expression may also be regulated via the strain responsive sec ond messenger CAMP (mediated by CREB1), which also influ ences klf4, cfos, calcium pathway and muscle related genes and rgs2 expression. RUNX2B appears as an intermediate TF that regulates expression of a key osteoblast regulator, $o s x$, together with KLF4 and AP1.

\section{Discussion}

Unravelling the molecular basis of phenotypic plasticity is a powerful first step in empirically evaluating the potential role of plasticity in evolution (West-Eberhard 2004, 2005; Bell \& Aubin-Horth 2010; Beldade et al. 2011). Indeed, molecular tools are increasingly incorporated into studies of plasticity in phenotypic traits such as melanization in Daphnia (Scoville \& Pfrender 2010), phase change in locusts (Wang \& Kang 2014), hypoxia tolerance in killifish (Whitehead et al. 2011) and changing reproductive phenotypes in cichlids (Maruska et al. 2011). Our study examines gene expression across a developmental time-course, shedding light on the transcriptional network involved with the initiation of phenotypic plasticity in the LPJ of the cichlid Astatoreochromis alluaudi. Drawing upon our previous transcriptome study (Gunter et al. 2013), 19 candidate genes were selected, with putative functions that span various organizational levels within the molecular network that orchestrates LPJ development. By examining the expression of these candidate genes in LPJ samples taken during the early divergence of diet-induced phenotypes, we gained insight into the complex molecular network that drives the development of a famous example of adaptive phenotypic plasticity.

This study is, to our knowledge, the first to use controlled experimental conditions to examine the developmental onset of diet-induced plasticity in the LPJs of A. alluaudi, identifying the period between 3 and 5 months of diet treatment as a critical period for the first appearance of measurable plasticity. At this stage, the SLs of both treatment and control fish were $\sim 55$ $60 \mathrm{~mm}$, consistent with previous observations on aquarium-raised $A$. alluaudi (Huysseune et al. 1994). This size at divergence is relatively delayed compared with the wild populations that inhabit Lake Victoria and its surrounding satellite lakes, for which LPJ divergence already occurs at an SL of $\sim 40 \mathrm{~mm}$ (Hoogerhoud 1986b). Our observation suggests that the applied treatment was less intensive than that provided by natural conditions, where juvenile fish may ingest hard food items at an earlier age and where their diet is likely to be more diverse than what we have provided (Cosandey-Godin et al. 2008). It is also plausible that selection may have acted upon the norm of reaction of this laboratory line, which has been bred in captivity for more than 25 years.

The candidate gene expression analyses indicated that major transcriptional changes coincide with the morphological transition period between 3 and 5 months of treatment. The first significant expression differences were detected between diet groups after 3 months of treatment, notably an upregulation of calcium pathway genes and immediate early genes amongst HD fish. Calcium channels are known to be rapidly activated in osteoblasts in response to mechanical strain (Walker et al. 2000), which in turn initiates the upregulation of immediate early genes (Chen et al. 2000). The combined upregulation of both calcium and immediate early genes after a treatment period of 3 months is consistent with our hypothesis that intense mechanical strain precedes the appearance of plastic phenotypes in A. alluaudi. A treatment period of 5 months was associated with a relative increase in expression of $o s x$, a major regulator of osteoblast differentiation, in HD fish. Notably, 5 months of treatment coincides with the first period of detectable morphological divergence, most probably involving enhanced proliferation of osteoblasts. Finally, we identified col12 to be significantly upregulated in HD fish after 8 months of treatment, which is likely to reflect alterations to the tensile properties of the extracellular matrix 
(Izu et al. 2011), concomitant with an overall increase in size of the LPJ. The majority of gene categories were relatively upregulated in HD at 3 months and beyond, albeit they infrequently attained statistical significance, likely due, in part, to our relatively low sample size. Nonetheless, it is interesting to note that statistically significant gene expression differences were observed in an order that reflects our hypothesis that mechanically responsive genes are initiated first, followed by osteoblast differentiation genes, then matrix-related genes (Gunter \& Meyer 2014 , in press).

The cluster analysis indicated a high degree of co-expression amongst genes of related functional categories, suggesting that they form regulatory modules that act in concert, generating the observed phenotypic plasticity. Three major regulatory clusters were identified by our analyses, including one comprised of the immediate early genes and klf4, a second that includes matrix- and bone-related genes, and a third that includes muscle-related and calcium pathway genes. The identified clusters comprise genes of multiple functional categories; however, it is likely that in this context, they are functionally related. For example, while klf4 is a pleiotropic transcription factor (Dang et al. 2000), its clustering with immediate early genes suggests that klf4 expression follows an immediate early pattern of induction in the A. alluaudi LPJ, similar to previous observations of its response to fluid shear stress (Peters et al. 2003). Moreover, the coregulation of bone- and matrix-related genes, as well as musclerelated and calcium pathway genes could be due to the fact that osteoblasts secrete bone matrix, and calcium flux is integral to muscle function.

Intriguingly, we observed differential expression of muscle genes in HD and SD LPJs, despite being cleaned of muscle prior to RNA extraction. This pattern is potentially due to the crosstalk between bone and muscle pathways (Mo et al. 2012; Bonewald et al. 2013). It has been demonstrated that muscle damage induces the differentiation of myoblastic precursors in the bone marrow cavities of mice, which migrate into the muscles and assist with their repair (Ferrari et al. 1998; LaBarge \& Blau 2002). Thus, the observed upregulation of muscle genes in HD jaws at 3 months may be induced by damage to the LPJ adductor muscles during chewing. The detected co-expression of calcium and muscle genes further suggests that myocytes undergo differentiation in the LPJ, as calcium homeostasis is integral to myogenesis and muscle contractile function (Davies 1963; Hauser et al. 2008).

In order to further characterize the regulatory relationships amongst our candidate genes, the transcription factor binding sites in their promoter regions were analysed. Specifically, we sought to identify genes whose expression is likely to respond to our diet treatments, first focusing on two mechanically responsive transcription factors, AP1 and CREB1 (cAMP response element-binding protein 1) (Davidovitch et al. 1984; Nomura \& Takano-Yamamoto 2000), as mechanical strain is the environmental stimulus that induces plasticity in A. alluaudi (Gunter et al. 2013). AP1 binding sites were detected in the promoter regions of all 19 candidate genes, and CREB1 binding sites were detected in 12 of the 19 candidates. This abundance of TFBSs suggests that immediate early genes such as AP1 are major regulators of phenotypic plasticity in the cichlid LPJ, influencing gene expression at various levels of the regulatory cascade (Fig. 7). Together, these observations and the detection of divergence in expression patterns prior to the onset of detectable morphological plasticity suggest that all genes in our analysis contribute to the establishment of the plastic phenotypes. However, constructing a structured regulatory hierarchy was beyond the scope of this project.

While we identified informative patterns of expression for most of our candidate genes, several genes displayed unexpected expression patterns, including some that were inverted in comparison with the results of our previous study (Gunter et al. 2013). Specifically, these include representatives from the immediate early, muscle-related and calcium pathway genes. We suggest that the dynamic expression of these genes reflects heterologous cycles of bone remodelling and tooth replacement that occur in the LPJ. In response to microdamage caused by mechanical strain, bones are locally resorbed by osteoclasts, and new, stronger bone is subsequently secreted by osteoblasts, improving the mechanical robustness of the bone (Hadjidakis \& Androulakis 2006). Indeed, many expression patterns induced in bones by mechanical strain vary considerably with time (Mantila Roosa et al. 2011). Remodelling is also an essential process in tooth replacement, which occurs approximately once per month in A. alluaudi (Huysseune 1995). Such dynamic gene expression patterns highlight the importance of including multiple developmental time points when trying to determine the molecular basis of phenotypic plasticity, as gene expression at a specific time point within a gene expression cycle may vary considerably. Moreover, we detected different expression patterns after 1 month of treatment, in comparison with 3,5 or 8 months, with the majority of candidate genes being upregulated in SD (albeit not significantly). We hypothesize that this may be due to a stressful period of adjustment to the mechanically stimulating diet that caused HD individuals to receive a lower level of nutrition than SD. It is also possible that gene expression after 1 month of treatment reflects an early stage of the plastic response, 
characterized by an overall decrease in gene expression, potentially representing an innate cost of plasticity. However, further experiments that include a pretreatment sample and denser sampling earlier in the development of plasticity are required to investigate this phenomenon in more detail.

Our study demonstrates that environmental inputs can act on a developmental pathway at various hierarchical levels, generating an adaptive and functionally integrated trait. Nineteen candidate plasticity genes, which encode a combination of transcription factors, signalling and structural proteins, were found to contain putative binding sites for mechanically responsive TFs in their promoter regions. Moreover, trends towards differential expression of these genes were observed prior to the appearance of plastic phenotypes. In particular, muscle and calcium genes displayed tight co-expression, suggesting that mechanical strain induces the differentiation of myocytes in the LPJ, integrating the development of these two tissue types. Together, these results suggest that diet-induced mechanical strain directly influences gene expression across various pathways, which together result in the development of a functionally integrated phenotype, such as the PJA of cichlid fishes. This finding extends the hypothesis of Young (2013) that the molecular pathways underlying phenotypic plasticity involve environmental stimulation of 'upstream genes', which direct the acquisition of a plastic phenotype, further altering the expression of 'downstream genes'. Specifically, our results indicate that the mechanically responsive TFs AP1 and CREB1 can regulate the expression of a suite of LPJ development genes without signalling via intermediates. Similarly, in the sea urchin, thermal stress was shown to influence the expression of genes distributed throughout the endomesodermal and ectomesodermal developmental network in a complex pattern that was not consistent with sequential gene regulation (Runcie et al. 2012).

The molariform phenotype of durophagous cichlids such as A. alluaudi is complex, involving coordinated alterations in the size of the pharyngeal jaws and their adductor muscles alongside an increase in tooth size and decrease in tooth number (Hoogerhoud 1986a; Huysseune et al. 1994; Huysseune 1995). Mismatch in any of these elements would render individuals less able to process hard food items, particularly in the light of the tight architectonic constraints within the oral cavity of teleosts (Meyer 1989; Smits et al. 1996; Chapman et al. 2001; Binning et al. 2010). Our previous investigation proposed that pleiotropic genes are likely to guide the development of both teeth and jaws (Gunter et al. 2013). Here, we propose a further molecular explanation for integrated development of muscles and bones of the PJA, namely through coordinated signalling between both tissues, which induces the differentiation of muscle precursor cells in the medullary cavity. Plasticity favours the development of complex, functionally integrated phenotypes without relying on multiple interdependent mutations, pleiotropy or linkage disequilibrium (West-Eberhard 2004, 2005). These investigations provide strong molecular evidence for the integration of different developmental modules during the establishment of adaptive phenotypes, which has previously been demonstrated on a morphological level (Badyaev et al. 2005).

In conclusion, through examining the expression of putative plasticity genes in a developmental context, we have identified that a strongly environmentally responsive network underlies adaptive plasticity in a key innovation of a cichlid fish. Our analysis identified the coordinated coexpression of functionally related genes, which we consider to represent regulatory modules. As mechanical strain directs the expression of each of these modules, it promotes the development of an integrated, complex phenotype, the molariform PJA, which enables the efficient exploitation of an alternative trophic niche in A. alluaudi. This study forms a platform to empirically assess the molecular trajectories that underlie adaptive phenotypes across a phylogenetic context in cichlid fishes. Ultimately, it will help to evaluate the importance of phenotypic plasticity in cichlid evolution, specifically in the light of evolutionary mechanisms such as genetic assimilation and genetic accommodation (Waddington 1953; West-Eberhard 2004).

\section{Acknowledgements}

We gratefully acknowledge financial support from the Deut sche Forschungsgemeinschaft (GU 1160/2 1) (HG), the Univer sity of Konstanz (AM), by the Zukunftskolleg at the University of Konstanz for HG and the China Scholarship Council for YL. We like to thank Xiong Fan for support in the laboratory, Car melo Fruciano for assistance with morphometric analyses and Patrick Niemantsverdriet and the late Dr. Frans Witte from the University of Leiden for generously providing their strain of A. alluaudi.

\section{References}

Abouheif E, Wray GA (2002) Evolution of the gene network underlying wing polyphenism in ants. Science, 297, 249252.

Adler FR, Harvell DC (1990) Inducible defenses, phenotypic variability and biotic environments. Trends in Ecology E Evo lution, 5, 407410.

An G, Miner CA, Nixon JC et al. (2010) Loss of Bright/ARID3a function promotes developmental plasticity. Stem Cells, 28, 15601567.

Aubin Horth N, Renn SC (2009) Genomic reaction norms: using integrative biology to understand molecular mecha 
nisms of phenotypic plasticity. Molecular Ecology, 18, 3763 3780 .

Badyaev AV, Foresman KR, Young RL (2005) Evolution of morphological integration: developmental accommodation of stress induced variation. The American Naturalist, 166, 382 395.

Beldade P, Mateus ARA, Keller RA (2011) Evolution and molecular mechanisms of adaptive developmental plasticity. Molecular Ecology, 20, 13471363.

Bell AM, Aubin Horth N (2010) What can whole genome expression data tell us about the ecology and evolution of personality? Philosophical Transactions of the Royal Society B: Biological Sciences, 365, 40014012.

Bergwerff M, Groot GD, Adriana C et al. (1998) Patterns of paired related homeobox genes PRX1 and PRX2 suggest involvement in matrix modulation in the developing chick vascular system. Developmental Dynamics, 213, 5970.

Binning S, Chapman L, Dumont J (2010) Feeding and breath ing: trait correlations in an African cichlid fish. Journal of Zoology, 282, 140149.

Bonewald L, Kiel D, Clemens T et al. (2013) Forum on bone and skeletal muscle interactions: summary of the proceed ings of an ASBMR workshop. Journal of Bone and Mineral Research, 28, 18571865.

Chapman L, Galis F, Shinn J (2001) Phenotypic plasticity and the possible role of genetic assimilation: Hypoxia, Aêinduced trade, Aêoffs in the morphological traits of an African cich lid. Ecology Letters, 3, 387393.

Chen NX, Ryder KD, Pavalko FM et al. (2000) Ca2+ regulates fluid shear induced cytoskeletal reorganization and gene expression in osteoblasts. American Journal of Physiology Cell Physiology, 278, C989 C997.

Clausen J, Keck DD, Hiesey WM (1941) Regional differentiation in plant species. American Naturalist, 75, 231 250.

Cosandey Godin A, Binning SA, Chapman LJ (2008) Special ized morphology for a non specialized diet: Liem's paradox in an African cichlid fish. McGill Science Undergraduate Research Journal, 3, 1923.

Dang DT, Pevsner J, Yang VW (2000) The biology of the mam malian Kruppel like family of transcription factors. The Inter national Journal of Biochemistry \& Cell Biology, 32, 11031121.

Davidovitch Z, Shanfeld J, Montgomery P et al. (1984) Bio chemical mediators of the effects of mechanical forces and electric currents on mineralized tissues. Calcified Tissue Inter national, 36, S86 S97.

Davies R (1963) A molecular theory of muscle contraction: cal cium dependent contractions with hydrogen bond formation plus ATP dependent extensions of part of the myosin actin cross bridges. Nature, 199, 10681074.

Denver RJ (1997) Environmental stress as a developmental cue: corticotropin releasing hormone is a proximate mediator of adaptive phenotypic plasticity in amphibian metamorphosis. Hormones and Behavior, 31, 169179.

Edidin M (1983) MHC antigens and non immune functions. Immunology Today, 4, 269270.

Elmer KR, Reggio C, Wirth T et al. (2009) Pleistocene desicca tion in East Africa bottlenecked but did not extirpate the adaptive radiation of Lake Victoria haplochromine cichlid fishes. Proceedings of the National Academy of Sciences, 106, 1340413409 .
Ferrari G, Angelis D, Coletta M et al. (1998) Muscle regenera tion by bone marrow derived myogenic progenitors. Science, 279, 15281530.

Gelse K, Poschl E, Aigner T (2003) Collagens structure, func tion, and biosynthesis. Advanced Drug Delivery Reviews, 55, 15311546.

Gilbert SF (2001) Ecological developmental biology: develop mental biology meets the real world. Developmental Biology, 233, 112.

Gilbert SF (2005) Mechanisms for the environmental regulation of gene expression: ecological aspects of animal develop ment. Journal of Biosciences, 30, 6574.

Gilbert SF, Epel D (2009) Ecological Developmental Biology. Sina uer Associates, Sunderland.

Greenwood P (1964) Environmental effects on the pharyngeal mill of a cichlid fish, Astatoreochromis alluaudi, and their taxo nomic implications. Proceedings of the Linnean Society of Lon don, 176, 110.

Greibe E, Fedosov S, Nexo E (2012) The cobalamin binding protein in zebrafish is an intermediate between the three cobalamin binding proteins in human. PLoS ONE, 7, e35660.

Gunter H, Meyer A (2014) Molecular investigation of mechani cal strain induced phenotypic plasticity in an acellular bone. Journal of Applied Ichthyology, 30, 630635.

Gunter HM, Fan S, Xiong F et al. (2013) Shaping development through mechanical strain: the transcriptional basis of diet induced phenotypic plasticity in a cichlid fish. Molecular Ecology, 22, 45164531

Haas BJ, Papanicolaou A, Yassour M et al. (2013) De novo tran script sequence reconstruction from RNA seq using the Trin ity platform for reference generation and analysis. Nature Protocols, 8, 14941512.

Hadjidakis DJ, Androulakis II (2006) Bone remodeling. Annals of the New York Academy of Sciences, 1092, 385396.

Hallikas O, Palin K, Sinjushina N et al. (2006) Genome wide prediction of mammalian enhancers based on analysis of transcription factor binding affinity. Cell, 124, 4759.

Hauser J, Saarikettu J, Grundstrom T (2008) Calcium regulation of myogenesis by differential calmodulin inhibition of basic helix loop helix transcription factors. Molecular Biology of the Cell, 19, 25092519.

Hoogerhoud R (1984) A taxonomic reconsideration of the ha plochromine genera Gaurochromis Greenwood, 1980 and Labrochromis Regan, 1920 (Pisces, Cichlidae). Netherlands Journal of Zoology, 34, 539565.

Hoogerhoud R (1986a) The adverse effects of shell ingestion for molluscivorous cichlids, a constructional morphological approach. Netherlands Journal of Zoology, 37, 34.

Hoogerhoud RJC (1986b) Ecological Morphology of Some Cichlid Fishes. RJC Hoogerhoud.

Hulsey CD (2006) Function of a key morphological innovation: fusion of the cichlid pharyngeal jaw. Proceedings of the Royal Society B: Biological Sciences, 273, 669675.

Huysseune A (1995) Phenotypic plasticity in the lower pharyn geal jaw dentition of Astatoreochromis alluaudi (Teleostei: Cic hlidae). Archives of Oral Biology, 40, 10051014.

Huysseune A, Sire J, Meunier F (1994) Comparative study of lower pharyngeal jaw structure in two phenotypes of Astatoreochromis alluaudi (Teleostei: Cichlidae). Journal of Mor phology, 221, 2543. 
Izu Y, Sun M, Zwolanek D et al. (2011) Type XII collagen regu lates osteoblast polarity and communication during bone for mation. The Journal of Cell Biology, 193, 11151130.

Jablonka E, Raz G (2009) Transgenerational epigenetic inheri tance: prevalence, mechanisms, and implications for the study of heredity and evolution. The Quarterly Review of Biol ogy, 84, 131176.

Janzen FJ, Paukstis GL (1991) Environmental sex determination in reptiles: ecology, evolution, and experimental design. Quarterly Review of Biology, 66, 149179.

Karttunen JT, Lehner PJ, Gupta SS, Hewitt EW, Cresswell P (2001) Distinct functions and cooperative interaction of the subunits of the transporter associated with antigen process ing (TAP). Proceedings of the National Academy of Sciences, 98, 74317436

Kocher TD, Conroy JA, McKaye KR, Stauffer JR (1993) Similar morphologies of cichlid fish in Lakes Tanganyika and Malawi are due to convergence. Molecular Phylogenetics and Evolution, 2, 158165.

Kwon AT, Arenillas DJ, Hunt RW, Wasserman WW (2012) oPOSSUM 3: advanced analysis of regulatory motif over rep resentation across genes or ChIP Seq datasets. G3: Genes Ge nomes Genetics, 2, 9871002.

LaBarge MA, Blau HM (2002) Biological progression from adult bone marrow to mononucleate muscle stem cell to multinucleate muscle fiber in response to injury. Cell, 111, 589601.

Lande R, Arnold SJ (1983) The measurement of selection on correlated characters. Evolution, 36, 12101226.

Landry CR, Aubin Horth N (eds) (2014) Recent advances in ecological genomics: from phenotypic plasticity to conver gent and adaptive evolution and speciation. In: Ecological Genomics pp. 1 5. Springer, Dordrecht, Netherlands.

Leberer E, Charuk J, Green NM, Maclennan DH (1989) Molecular cloning and expression of cDNA encoding a lumenal calcium binding glycoprotein from sarcoplasmic reticulum. Proceedings of the National Academy of Sciences, 86, 60476051.

Lerch JK, Kuo F, Motti D et al. (2012) Isoform diversity and regulation in peripheral and central neurons revealed through RNA Seq. PLoS ONE, 7, e30417.

Lewontin RC (1974) The Genetic Basis of Evolutionary Change. Columbia University Press, New York.

Li H, Handsaker B, Wysoker A et al. (2009a) The sequence alignment/map format and SAMtools. Bioinformatics, 25, 20782079

Li N, Felber K, Elks P, Croucher P, Roehl HH (2009b) Tracking gene expression during zebrafish osteoblast differentiation. Developmental Dynamics, 238, 459466.

Liem KF (1974) Evolutionary strategies and morphological innovations: cichlid pharyngeal jaws. Systematic Biology, 22, 425441.

Lu M F, Cheng H T, Kern MJ et al. (1999) prx 1 functions coop eratively with another paired related homeobox gene, prx 2, to maintain cell fates within the craniofacial mesenchyme. Development, 126, 495504.

Mantila Roosa SM, Liu Y, Turner CH (2011) Gene expression patterns in bone following mechanical loading. Journal of Bone and Mineral Research, 26, 100112.

Maruska KP, Levavi Sivan B, Biran J, Fernald RD (2011) Plas ticity of the reproductive axis caused by social status change in an African cichlid fish: I. pituitary gonadotropins. Endocri nology, 152, 281290.

Mayr E (1963) Animal Species and Evolution. Harvard Press, Cambridge, Massachusetts.

Mei J, Zhang Q Y, Li Z, Lin S, Gui J F (2008) C1q like inhibits p53 mediated apoptosis and controls normal hematopoiesis during zebrafish embryogenesis. Developmental Biology, 319, 273284 .

Meissner G (1994) Ryanodine receptor/Ca2+ release channels and their regulation by endogenous effectors. Annual Review of Physiology, 56, 485508.

Meyer A (1987) Phenotypic plasticity and heterochrony in Cic hlasoma managuense (Pisces, Chichlidae) and their implica tions for speciation in Cichlid fishes. Evolution, 41, 1357 1369.

Meyer A (1989) Cost of morphological specialization: feeding performance of the two morphs in the trophically polymor phic cichlid fish, Cichlasoma citrinellum. Oecologia, 80, 431 436.

Meyer A (1990) Morphometrics and allometry in the trophi cally polymorphic cichlid fish, Cichlasoma citrinellum: alterna tive adaptations and ontogenetic changes in shape. Journal of Zoology, 221, 237260.

Meyer A (1993a) Phylogenetic relationships and evolutionary processes in East African cichlid fishes. Trends in Ecology $\mathcal{E}$ Evolution, 8, 279284

Meyer A (1993b) Trophic polymorphisms in cichlid fish: do they represent intermediate steps during sympatric specia tion and explain their rapid adaptive radiation. Trends in Ich thyology, 7, 257266.

Meyer A, Kocher TD, Basasibwaki P, Wilson AC (1990) Monophyletic origin of Lake Victoria cichlid fishes suggested by mitochondrial DNA sequences. Nature, 347, 550553.

Michikami I, Fukushi T, Tanaka M et al. (2012) Kruppel like factor 4 regulates membranous and endochondral ossifica tion. Experimental Cell Research, 318, 311325.

Mo C, Romero Suarez S, Bonewald L, Johnson M, Brotto M (2012) Prostaglandin E2: from clinical applications to its potential role in bone muscle crosstalk and myogenic differ entiation. Recent Patents on Biotechnology, 6, 223.

Morgan L (1947) A variable phenotype associated with the fourth chromosome of Drosophila melanogaster and affected by heterochromatin. Genetics, 32, 200.

Muschick M, Barluenga M, Salzburger W, Meyer A (2011) Adaptive phenotypic plasticity in the Midas cichlid fish pha ryngeal jaw and its relevance in adaptive radiation. $B M C$ Evolutionary Biology, 11, 116.

Nakashima K, Zhou X, Kunkel G et al. (2002) The novel zinc finger containing transcription factor osterix is required for osteoblast differentiation and bone formation. Cell, 108, 17 29.

Nicotra AB, Atkin OK, Bonser SP et al. (2010) Plant phenotypic plasticity in a changing climate. Trends in Plant Science, 15, 684692.

Nomura S, Takano Yamamoto T (2000) Molecular events caused by mechanical stress in bone. Matrix Biology, 19, 91 96.

Nonaka MI, Nonaka M (2010) Evolutionary analysis of two classical MHC class I loci of the medaka fish, Oryzias latipes: haplotype specific genomic diversity, locus specific polymor 
phisms, and interlocus homogenization. Immunogenetics, 62, 319332.

Nose K, Shibanuma M (1994) Induction of early response genes by hypergravity in cultured mouse osteoblastic cells (MC3T3 E1). Experimental Cell Research, 211, 168170.

Ott CE, Bauer S, Manke T et al. (2009) Promiscuous and depo larization induced immediate early response genes are induced by mechanical strain of osteoblasts. Journal of Bone and Mineral Research, 24, 12471262.

Perry S (1998) Troponin T: genetics, properties and function. Journal of Muscle Research \& Cell Motility, 19, 575602.

Peters DG, Zhang X C, Benos PV, Heidrich O'Hare E, Ferrell RE (2003) Genomic analysis of immediate/early response to shear stress in human coronary artery endothelial cells. Phys iological Genomics, 12, 2534.

Pfennig DW, Wund MA, Snell Rood EC et al. (2010) Pheno typic plasticity's impacts on diversification and speciation. Trends in Ecology \& Evolution, 25, 459467.

Pigliucci M (2007) Do we need an extended evolutionary syn thesis? Evolution, 61, 27432749.

Price TD, Qvarnstrom A, Irwin DE (2003) The role of pheno typic plasticity in driving genetic evolution. Proceedings of the Royal Society of London. Series B: Biological Sciences, 270, 1433 1440.

RDC Team (2005) R: A Language and Environment for Statistical Computing. ISBN 3900051070 . R Foundation for Statistical Computing, Vienna, Austria, 2013.

Runcie DE, Garfield DA, Babbitt CC et al. (2012) Genetics of gene expression responses to temperature stress in a sea urchin gene network. Molecular Ecology, 21, 45474562.

Sadlon TJ, Dell'Oso T, Surinya KH, May BK (1999) Regulation of erythroid 5 aminolevulinate synthase expression during erythropoiesis. The International Journal of Biochemistry $\mathcal{E}$ Cell Biology, 31, 11531167

Salzburger W, Mack T, Verheyen E, Meyer A (2005) Out of Tanganyika: genesis, explosive speciation, key innovations and phylogeography of the haplochromine cichlid fishes. BMC Evolutionary Biology, 5, 17.

Scheiner SM, Holt RD (2012) The genetics of phenotypic plas ticity. X. Variation versus uncertainty. Ecology and Evolution, 2, 751767

Schlichting CD, Pigliucci M (1998) Phenotypic Evolution: A Reac tion Norm Perspective. Sinauer Associates Incorporated, Sun derland, Massachusetts.

Schon I, Martens K (2004) Adaptive, pre adaptive and non adaptive components of radiations in ancient lakes: a review. Organisms Diversity \& Evolution, 4, 137156.

Schwander T, Leimar O (2011) Genes as leaders and followers in evolution. Trends in Ecology \& Evolution, 26, 143151.

Scoville AG, Pfrender ME (2010) Phenotypic plasticity facilitates recurrent rapid adaptation to introduced predators. Proceedings of the National Academy of Sciences, 107, 42604263.

Sen B, Styner M, Xie Z et al. (2009) Mechanical loading regu lates NFATc1 and $\beta$ catenin signaling through a GSK3 $\beta$ con trol node. Journal of Biological Chemistry, 284, 3460734617.

Slootweg R, Malek E, McCullough F (1994) The biological con trol of snail intermediate hosts of schistosomiasis by fish. Reviews in Fish Biology and Fisheries, 4, 6790.

Smits J, Witte F, Veen F (1996) Functional changes in the anat omy of the pharyngeal jaw apparatus of Astatoreochromis al luaudi (Pisces, Cichlidae), and their effects on adjacent structures. Biological Journal of the Linnean Society, 59, 389 409.

Snell Rood EC, Van Dyken JD, Cruickshank T, Wade MJ, Moc zek AP (2010) Toward a population genetic framework of developmental evolution: the costs, limits, and consequences of phenotypic plasticity. BioEssays, 32, 7181.

Sommer RJ, Ogawa A (2011) Hormone signaling and pheno typic plasticity in nematode development and evolution. Current Biology, 21, R758 R766.

Song G, Harding SE, Duchen MR et al. (2002) Altered mechani cal properties and intracellular calcium signaling in cardio myocytes from annexin 6 null mutant mice. The FASEB Journal, 16, 622624.

Stiassny ML, Meyer A (1999) Cichlids of the rift lakes. Scientific American, 6469.

Sumpio BE, Du W, Xu W J (1994) Exposure of endothelial cells to cyclic strain induces $\mathrm{c}$ fos, fosB and $\mathrm{c}$ jun but not jun $\mathrm{B}$ or jun $\mathrm{D}$ and increases the transcription factor $\mathrm{AP} 1$. Endothe lium, 2, 149156.

Swynghedauw B (2006) Phenotypic plasticity of adult myocar dium: molecular mechanisms. Journal of Experimental Biology, 209, 23202327.

Thibert Plante X, Hendry A (2011) The consequences of pheno typic plasticity for ecological speciation. Journal of Evolution ary Biology, 24, 326342.

Treves S, Anderson AA, Ducreux S et al. (2005) Ryanodine receptor 1 mutations, dysregulation of calcium homeostasis and neuromuscular disorders. Neuromuscular Disorders, 15, 577587.

Tucker R, Hagios C, Chiquet Ehrismann R, Lawler J (1997) In situ localization of thrombospondin 1 and thrombospondin 3 transcripts in the avian embryo. Developmental Dynamics, 208, 326337.

Vandesompele J, De PreterK, Pattyn F et al. (2002) Accurate normalization of real time quantitative RT PCR data by geo metric averaging of multiple internal control genes. Genome Biology, 3, research0034.

Versele M, de Winde JH, Thevelein JM (1999) A novel regula tor of $\mathrm{G}$ protein signalling in yeast, Rgs2, downregulates glu cose activation of the cAMP pathway through direct inhibition of Gpa2. The EMBO Journal, 18, 55775591.

Waddington $\mathrm{CH}$ (1953) Genetic assimilation of an acquired character. Evolution, 7, 118126.

Walker L, Publicover S, Preston M, Said Ahmed M, El Haj A (2000) Calcium channel activation and matrix protein upreg ulation in bone cells in response to mechanical strain. Journal of Cellular Biochemistry, 79, 648661.

Wang X, Kang L (2014) Molecular mechanisms of phase change in locusts. Annual Review of Entomology, 59, 225244.

West Eberhard MJ (2004) Developmental Plasticity and Evolution. Oxford University Press, New York.

West Eberhard MJ (2005) Developmental plasticity and the origin of species differences. Proceedings of the National Academy of Sciences of the United States of America, 102, 65436549.

Whitehead A, Roach JL, Zhang S, Galvez F (2011) Genomic mechanisms of evolved physiological plasticity in killifish distributed along an environmental salinity gradient. Proceed ings of the National Academy of Sciences, 108, 61936198. 
Woltereck R (1913) Beitrag zur Analyse der „Vererbung erworbener Eigenschaften": transmutation und Prainduktion beiDaphnia. Molecular and General Genetics MGG, 9, 146155.

Wright WE, Sassoon DA, Lin VK (1989) Myogenin, a factor regulating myogenesis, has a domain homologous to MyoD. Cell, 56, 607617.

Wund MA, Baker JA, Clancy B, Golub JL, Foster SA (2008) A test of the flexible stem model of evolution: ancestral plastic ity, genetic accommodation, and morphological divergence in the threespine stickleback radiation. The American Natural ist, 172, 449462.

Young RL (2013) Linking conceptual mechanisms and tran scriptomic evidence of plasticity driven diversification. Mole cular Ecology, 22, 43634365.

Young KA, Snoeks J, Seehausen O (2009) Morphological diver sity and the roles of contingency, chance and determinism in African cichlid radiations. PLoS ONE, 4, e4740.

H.G. and A.M. conceived and designed the experiments; H.G. and R.S. conducted the experiments; R.S. and Y.L. performed the data analysis; R.S. and H.G. wrote the first draft of manuscript and all authors contributed to and approved the final version.

\section{Data accessibility}

Linear morphometric measurements: doi:10.5061/dryad. rc638

Normalized relative quantification (RQ) of candidate genes: doi:10.5061/dryad.rc638

Distance matrices of the cluster analyses: doi:10.5061/ dryad.rc638

\section{Supporting information}

Additional supporting information may be found in the online ver sion of this article.
Fig. S1 Gene clusters found in hierarchical cluster analyses of candidate genes coincide strongly for this and a previous study.

Fig. S2 Standard length of specimens according to treatment group and time in treatment.

Fig. S3 Residuals of linear morphometric measurements across time points and between diet groups.

Fig. S4 Linear morphometric measurements across SL of speci mens in the two diet groups.

Fig. S5 PCA on linear morphometric measurements suggests phenotypic divergence between diet groups.

Fig. S6 Mean gene expression between diet groups across the developmental time course. Solid and dashed lines reflect SD and HD fish, respectively. Error bars reflect 95\% CIs.

Fig. S7 Individual hierarchical cluster analyses for each of the three major gene cluster.

Table S1 Gene abbreviations and primers used in this study.

Table S2 Results of linear morphometric measurement com parisons between diet groups.

Table S3 ANCOVAS on linear morphometric measures consider ing diet groups, time point and SL of the fish.

Table S4 Comparisons of $R Q$ values between diet groups for each time point.

Table S5 Loadings and importance of components for the PCA on morphometric measurements (Fig. S5, Supporting Informa tion).

Table S6 Loadings and importance of components for the PCA on gene expression.

Table S7 Distribution of TFBSs in the promoters of candidate genes. 